\title{
Synthesis of High-Antifouling and Antibacterial Ultrafiltration Membranes Incorporating Low Concentrations of Graphene Oxide
}

\section{Abedalkader Alkhouzaam, Hazim Qiblawey, Majeda Khraisheh}

\begin{abstract}
Objectives
- NOx free and high-oxidation synthesis of graphene oxide $(\mathrm{GO})$ from natural graphite via modified Hummers' method.

Preparation of polysulfone (PSF) ultrafiltration membranes incorporating the synthesized GO.

- Testing the separation properties of the prepared membranes.

- Testing the fouling resistance of the prepared membranes against organic and protein fouling.

Testing the antibacterial activity of the GO-based membranes.

\section{Overview \\ Membrane treatment for wastewater treatment is one of the promising solutions to affordable} clean water. It is a developing technology throughout the world and considered as the most effective and economical method available. However, the limitations of membranes' mechanical and chemical properties restrict their industrial applications. Fouling is a process where contaminants in feed water deposit onto membrane surface or within the membrane pores, consequently causing flux decline and lowering the permeate quality. Therefore, developing antifouling membranes and finding new antifouling agents have become an important research objective.

One of the recently investigated nanomaterials in membrane science for water treatment and desalination is graphene oxides (GO). Because of its high mechanical strength easy accessibility, and chemical stabilities, GO was considered as one of the promising fillers that can reduce the fouling of membranes while enhancing their performance with respect to water flux and salt rejection
\end{abstract}

\section{Methodology}

- Synthesis of graphene oxide (GO): High-oxidation and $\mathrm{NO}_{x}$ free synthesis of GO was conducted using modified Hummers' method. Graphite (1 g)

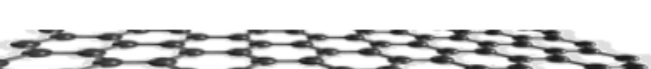

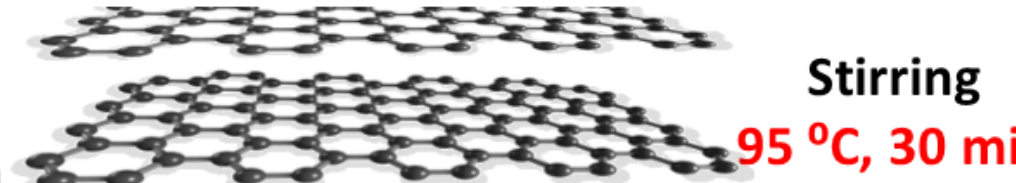

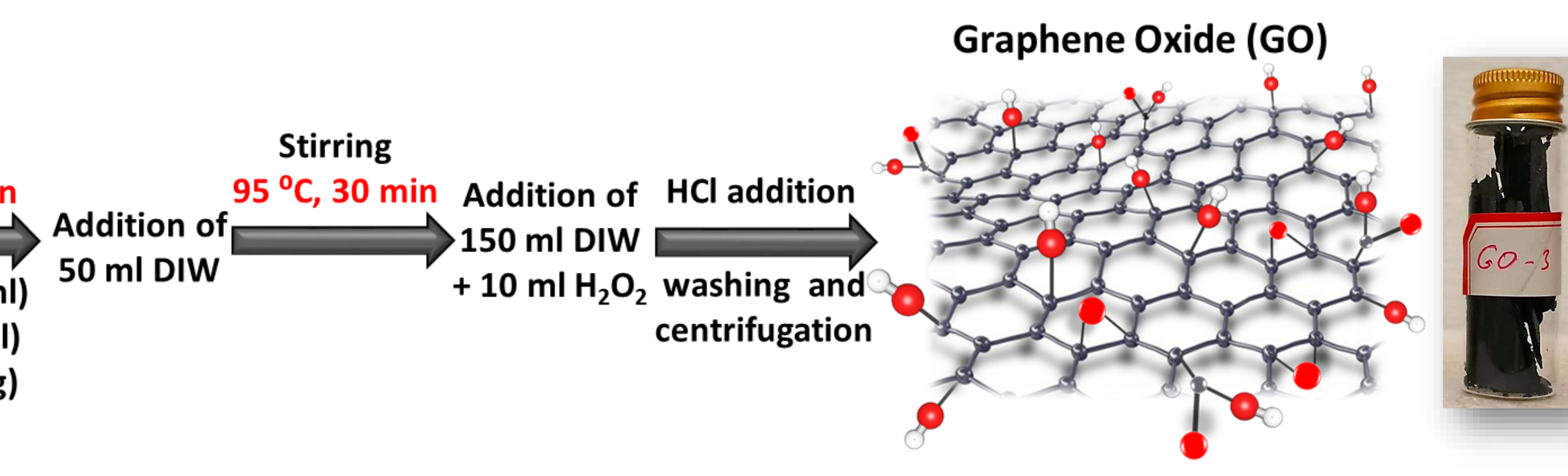

Membranes preparation: Pristine polysulfone (PSF) and GO-PSF composite membranes were prepared via phase inversion technique.

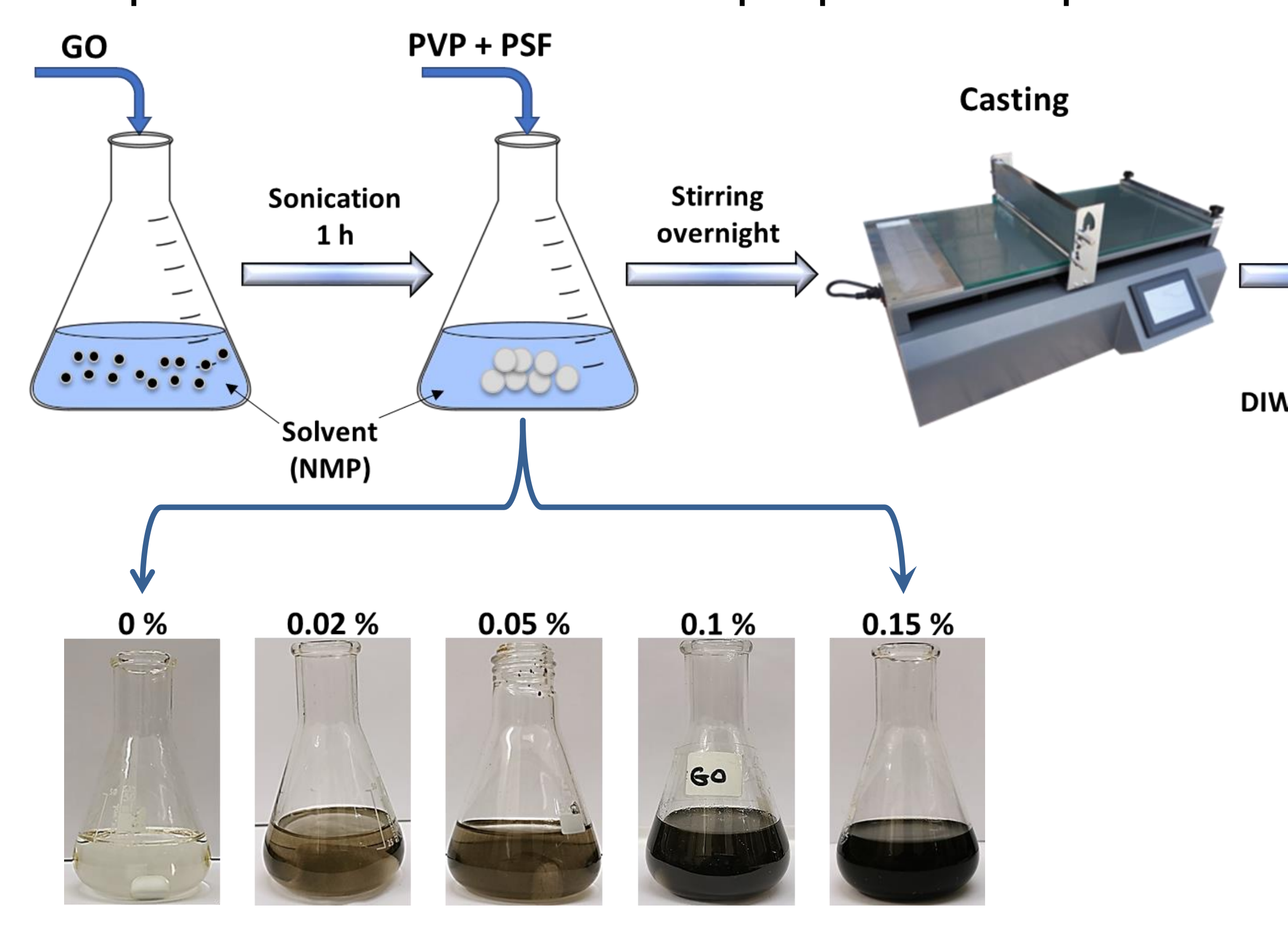

Separation, flux, and antifouling measurements:

- Experiments were conducted using crossflow membrane unit.

- Two model foulants were used, BSA and $H A$, representing protein and organic fouling.

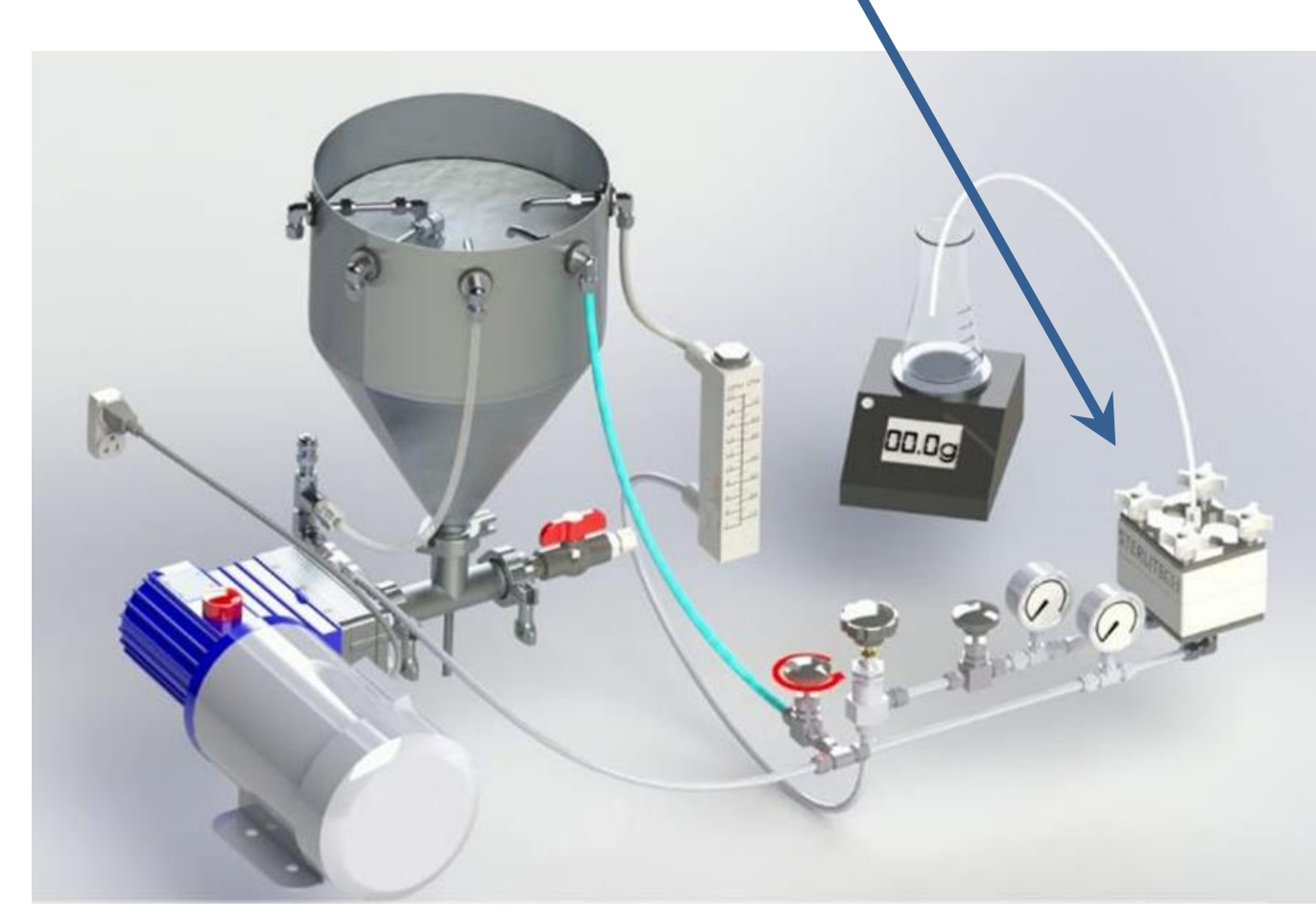

- Antibacterial activity test: The antibacterial actitivy of the prepared membranes were investigated by bacteriostasis rate determination using Halomonas aquamarina as the model bacterium

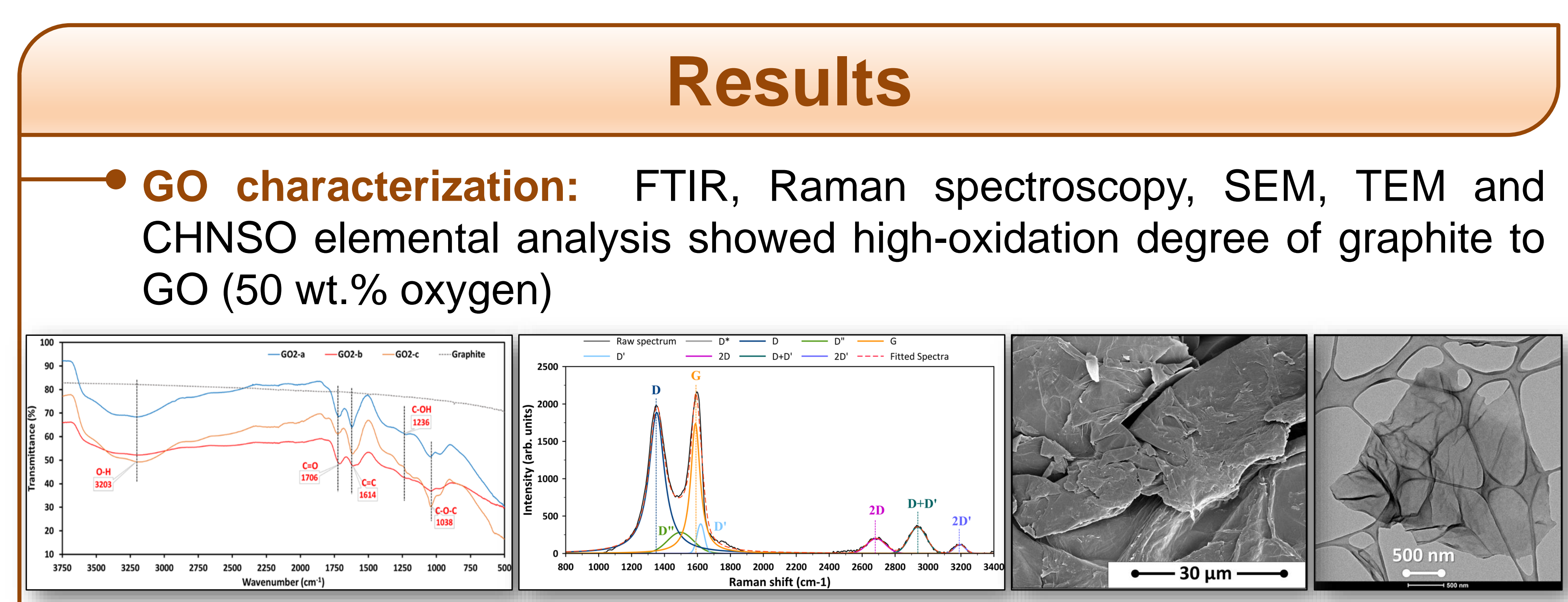

Membranes characterization:

- SEM images showed well dispersion of GO particles in the PSF matrix.

- Surface roughness increased with GO addition.

- Pore size decreased with $\mathrm{GO}$ addition due to the agglomeration of GO in the pores.

- Hydrophilicity increased with GO addition.

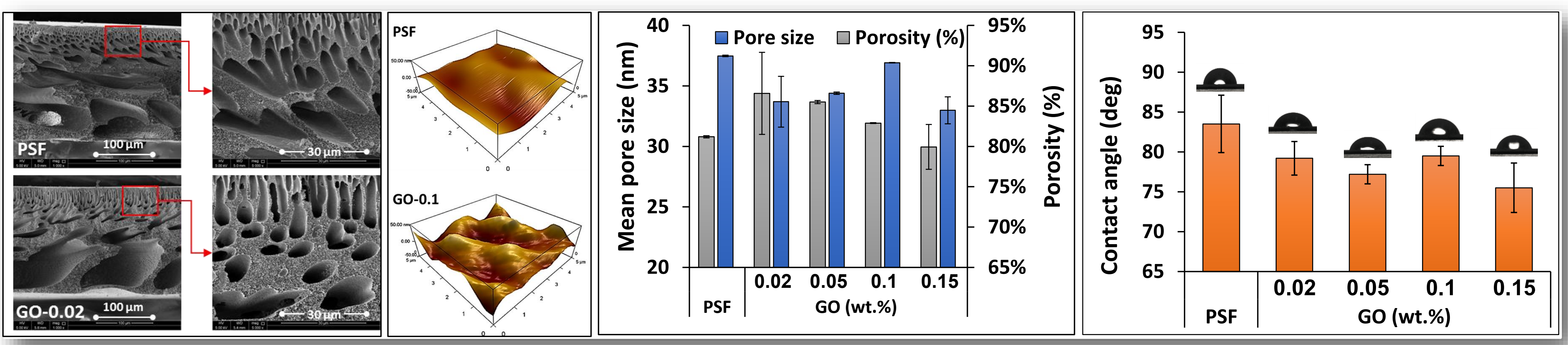

- Separation properties:

- All membranes showed complete rejection of BSA and HA.

- Flux decreased with GO addition.

- Fouling resistance, represented by flux recovery ratio (FRR), was significantly improved with $\mathrm{GO}$ addition.

- FRR of GO-based membranes increased up to $86.9 \%$ and $97 \%$ for BSA and HA respectively.

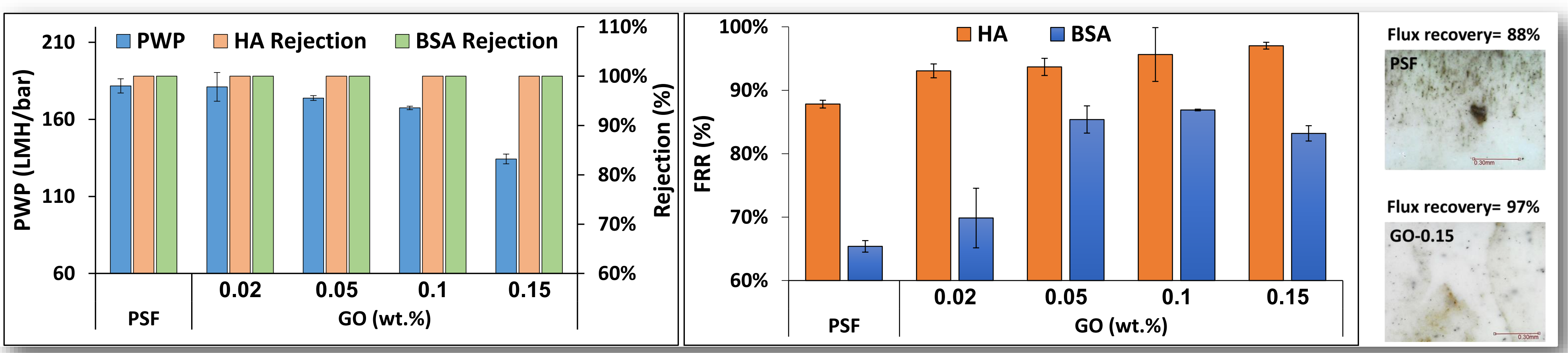

- Antibacterial activity: membranes with 0.15 wt.\% GO exhibited a bacteriostatis rate of 62.9 $\%$ against Halomonas.
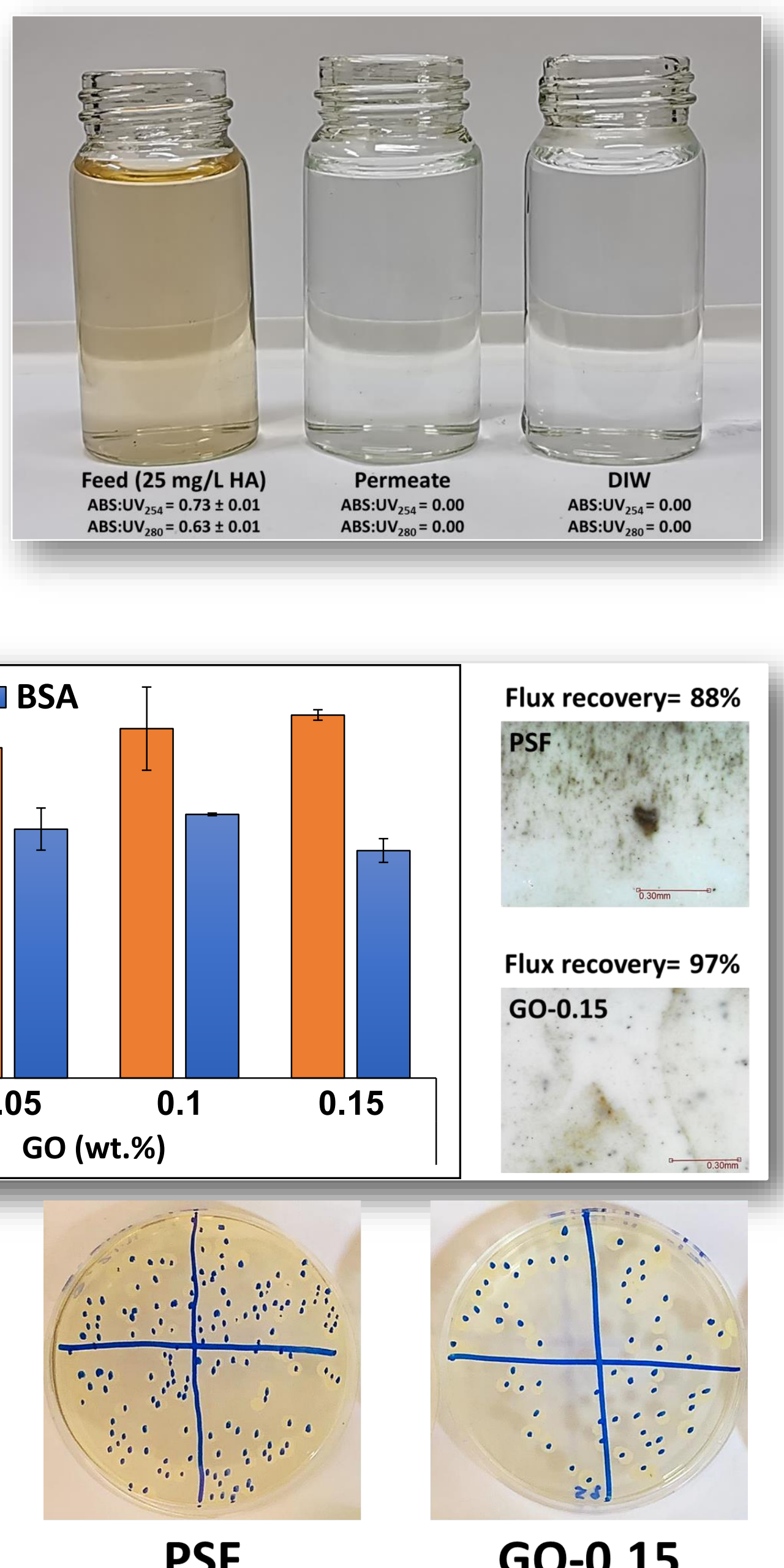

\section{Conclusion}

- The development of antifouling and antibacterial membranes has become one of the well-investigated objectives in the past years.

- Pristine polymers commonly exhibit a reduction in flux over the time due to the deposition of foulant molecules on the surface or within the membrane pores.

- GO is one of the promising nanofillers that can enhance separation antifouling properties.

- Flux was found to be reduced with high concentrations of $\mathrm{GO}$ due to the agglomeration of $\mathrm{GO}$ in the pores.

- All prepared membranes exhibit complete rejection of protein and humic acids.

- GO showed significant enhancement of fouling resistance against protein and organic fouling.

- GO was found to have good antibacterial activity which enhanced the bacteriostatis rate of GO-based membranes compared to pristine PSF.

\section{Acknowledgment}

This work was made possible by an Award [GSRA4-1-0504-17043] from Qatar National Research Fund (QNRF). The contents herein are solely the responsibility of the authors. Authors would like to thank also the Central Lab Unit (CLU) in Qatar University (QU) for their support in GO characterization. 